Pacific Journal of Mathematic 


\section{SEMI-ORTHOGONALITY IN RICKART RINGS}

\section{LOUIS HERMAN}

This note initiates a study of the semi-orthogonality relation on the lattice of principal left ideals generated by idempotents of a Rickart ring. It will be seen that two left ideals in a von Neumann algebra are semi-orthogonal if and only if their unique generating projections are non-asymptotic. Connections between semi-orthogonality, dual modularity, von Neumann regularity, and algebraic equivalence will be established; those Rickart rings with a superabundance of semiorthogonal left ideals will be characterized.

A regular ring is a ring $A$ with identity in which each element $a \in A$ is regular in the sense that $a b a=a$ for some element $b \in A$. A Rickart ring is a ring $A$ with identity in which the left (and right) annihilator of each element is a principal left (right) ideal generated by an idempotent. Regular rings and Baer rings, as defined by Kaplansky [4], are special cases of Rickart rings: in particular, then, a von Neumann algebra is a Rickart ring. Rickart rings are called Baer rings in [2]. Throughout this note, $A$ will denote a Rickart ring. $L(M)$ and $R(M)$ will denote respectively the left and right annihilators of a subset $M$ of $A$. The letters $e, f, g, h$ and $k$ will denote idempotents and the letters $E, F, G, H$ and $K$ will denote the left ideals they generate.

Ordered by set inclusion, the set $L(A)$ of principal left ideals generated by idempotents forms a lattice. If $E$ and $F$ form a modular pair in $\boldsymbol{L}(A)$, we shall write $(E, F) M$; if $E$ and $F$ form a dual modular pair in $L(A)$, we shall write $(E, F) M^{*}$. Following S. Maeda [6], we shall say that two left ideals $E$ and $F$ in $\boldsymbol{L}(A)$ are semi-orthogonal, $E \# F$, if they are generated by orthogonal idempotents. Maeda shows that the semi-orthogonality relation $\#$ on $\boldsymbol{L}(A)$ has these properties: (1) If $E \# E$, then $E=(0)$; (2) If $E \# F$, then $F \# E$; (3) If $E_{1} \leqq E$ and $E \# F$, then $E_{1} \# F$; (4) If $E \# F$ and $E \vee F \# G$, then $E \# F \vee G$; (5) If $E \leqq F$, then there is a left ideal $G$ in $L(A)$ such that $E \vee G=F$ and $E \# G$.

The results herein form a portion of the author's dissertation, submitted to the Graduate School of the University of Massachusetts and directed by Professor D. J. Foulis.

2. Semi-orthogonal left ideals. In this section, we give geometric meaning to Maeda's canonical semi-orthogonality relation in $\boldsymbol{L}(A)$. 
Theorem 1. Let $E=A e$ and $F=A f$. Then the following conditions are equivalent:

(1) $E \# F$.

(2) $E \cap F=(0)$ and $e(1-f)$ is regular in $A$.

(3) $E \oplus F=E \vee F$ in $L(A)$.

Proof. The proofs of (1) implies (2) and of (3) implies (1) are routine. To see that (2) implies (3), we suppose that $e(1-f) x e(1-$ $f)=e(1-f)$ for some $x \in A$. Put $g=(1-f) x e(1-f)$. Then $f g=$ $0=g f$ and $e g=e(1-f) x e(1-f)=e(1-f)=e-e f$. Then $g^{2}=$ $(1-f) x e(1-f) g=(1-f) x e g=(1-f) x e(1-f)=g$ and $(f+g)^{2}=$ $f+f g+g f+g=f+g$.

We claim that $E \oplus F=A(f+g)$. But $f=(f+g)-g(f+g) \epsilon$ $A(f+g)$ and $e=e f+e g=e(f+g) \in A(f+g)$. Thus $E \oplus F \leqq A(f+$ g). Conversely, $f+g=f+(1-f) x e(1-f)=(1-f) x e+(1-x e+$ $f x e) f \in E \oplus F$. Hence $E \oplus F=A(f+g) \in L(A)$.

We can find perspicacious geometric and topological interpretations for each of these equivalent conditions in the ring of bounded operators on a Hilbert space or, more generally, in any von Neumann algebra. In such a ring, any left annihilator is a principal left ideal generated by a unique projection (= self-adjoint idempotent). Let $e$ and $f$ denote the unique generating projections of $E$ and $F$ respectively: we shall identify these projections with their ranges.

If $e \wedge f=0, e$ and $f$ are said to be asymptotic if $\sup |\langle\alpha, \beta\rangle|=$ 1 , where $\|\alpha\|=1=\|\beta\|, \alpha \in e, \beta \in f$; otherwise $e$ and $f$ are said to be non-asymptotic. It is known [5, p. 166 and pp. 172-174] that these conditions are equivalent: (1) $e$ and $f$ form a non-asymptotic pair; (2) The projection map of the subspace $e \oplus f$ onto $e$ is continuous; (3) The vector sum of $e$ and $f$ is a closed subspace; (4) $(e, f) M^{*}$ in the projection lattice of the ring of all bounded operators on the underlying Hilbert space. The relation of semi-orthogonality to non-asymptoticity is provocative; for, by modifying results of Jacob Feldman [1, pp. 1214 ], it is easy to verify that $E \# F$ if and only if $e$ and $f$ form a nonasymptotic pair.

Our next result, though appearing an immediate consequence of Theorem 1 (2), seems to require a measure of prestidigitatorial skill with idempotents.

COROLLARY 1. ef is regular if and only if $(1-f)(1-e)$ is regular.

Proof. We prefer to demonstrate the obviously equivalent statement: If $e(1-f)$ is regular, then so is $f(1-e)$. To this end, choose 
an idempotent $h$ with $A h=A e \cap A f$. Put $e_{1}=e+h-e h$ and $f_{1}=$ $f+h-f h$. Then $e_{1}$ and $f_{1}$ are idempotent generators for $A e$ and Af respectively and $h=h e_{1}=e_{1} h=h f_{1}=f_{1} h$. By direct computation, we have $e_{1}\left(1-f_{1}\right)=e(1-f)(1-h)$ and $f_{1}\left(1-e_{1}\right)=f(1-e)(1-h)$. Since $e(1-f)$ is regular, $e(1-f) x e(1-f)=e(1-f)$ for some $x \in A$. Then, an easy computation shows $e_{1}\left(1-f_{1}\right)[(1-f) x] e_{1}(1-f)=e_{1}\left(1-f_{1}\right)$; thus $e_{1}\left(1-f_{1}\right)$ is regular.

Put $e_{0}=e_{1}(1-h)$ and $f_{0}=f_{1}(1-h)$. Then $e_{0}\left(1-f_{0}\right)=e_{1}\left(1-f_{1}\right)$ is regular. Moreover, if $z \in A e_{0} \cap A f_{0} \leqq A e_{1} \cap A f_{1}=A h$, then $z=z h$ $\left(z e_{0}\right) h=z e_{1}(1-h) h=0$; so $A e_{0} \cap A f_{0}=(0)$. Then by Theorem 1 (2), we have $A e_{0} \# A f_{0}$.

Consequently, $f(1-e)(1-h)=f_{1}\left(1-e_{1}\right)=f_{0}\left(1-e_{0}\right)$ is regular. Then $f(1-e)(1-h) y f(1-e)(1-h)=f(1-e)(1-h)$ for some element $y \in A$. But this means that $f(1-e)(1-h) y f(1-e)-f(1-e)=$ $f(1-e)(1-h) y f(1-e) h-f(1-e) h$ is an element of $A(1-e) \cap A h=$ $A(1-e) \cap A e \cap A f=(0)$. Thus $f(1-e)[(1-h) y] f(1-e)=f(1-e)(1-$ h) $y f(1-e)=f(1-e)$, showing that $f(1-e)$ is regular in $A$.

Corollary 2. If $E \# F$, then $(E, F) M$ and $(E, F) M^{*}$ in $L(A)$.

Proof. A proof that $E$ and $F$ form a modular pair is given by Maeda [6, Lm. 1]. Now suppose that $A e \# A f$ with $A f \leqq A g \leqq A e \oplus$ $A f$. Then $g=x e+y f$ for some elements $x$ and $y$ in $A$. Then $x e=$ $g-y f \in A e \cap A g$ and we have $g=x e+y f \in(A e \cap A g) \oplus A f$. Thus $A g \leqq(A e \cap A g) \oplus A f$. Since the opposite inclusion is evident, $A g=$ $(A e \cap A g) \oplus A f$. Hence $(A e, A f) M^{*}$.

3. Equivalence of left ideals. Two left ideals $E$ and $F$ in $L(A)$ are semi-orthogonally perspective via $G, G: E \sim F$, if $E \oplus G=E \vee F=$ $G \oplus F$ with $E \# G$ and $G \# F$. The importance of this relation is exemplified in the following result:

THEOREm 1. If $G: E \sim F$, then the mapping $E_{0} \rightarrow \varphi\left(E_{0}\right)=\left(E_{0} \oplus\right.$ $G) \cap F$ is a lattice isomorphism of the principal lattice ideal generated by $E$ in $L(A)$ onto the principal lattice ideal generated by $F$ in $L(A)$. Under this mapping, moreover, semi-orthogonal left ideals contained in $E$ correspond with semi-orthogonal left ideals contained in $F$.

Proof. The proof is entirely lattice theoretic. Define a mapping $\psi$ by $F_{0} \rightarrow\left(G \oplus F_{0}\right) \cap E$ for each $F_{0} \leqq F$; clearly both $\varphi$ and $\psi$ are isotone maps. By Corollary 2.2, we have $(F, G) M^{*}$ and $(G, E) M$. With these modularity relations, it is easy to compute $(\psi \circ \varphi)\left(E_{0}\right)=$ $E_{0}$ for all $E_{0} \leqq E$. Similarly $(\varphi \circ \varphi)\left(F_{0}\right)=F_{0}$ for all $F_{0} \leqq F$. Thus $\varphi$ is a lattice isomorphism with $\psi$ its inverse mapping. 
Now suppose $E_{1}, E_{2} \leqq E$ with $E_{1} \# E_{2}$. Since $E \# G, E_{1} \oplus E_{2} \# G$ also. Then $E_{1} \oplus G \# E_{2}$ and we may compute $\varphi\left(E_{1}\right) \oplus G=\left[\left(E_{1} \oplus G\right) \cap\right.$ $F] \oplus G=\left(E_{1} \oplus G\right) \cap(F \oplus G)=\left(E_{1} \oplus G\right) \cap(E \oplus G)=E_{1} \oplus G \# E_{2}$, since $(F, G) M^{*}$. Thus $\varphi\left(E_{1}\right) \# E_{2} \oplus G$, so that $\varphi\left(E_{1}\right) \# \varphi\left(E_{2}\right)$. Conversely, if $F_{1}, F_{2} \leqq F$ with $F_{1} \# F_{2}$, a similar argument shows $\psi\left(F_{1}\right) \# \psi\left(F_{2}\right)$.

Lemma 1. [7, Th. 2]. Let $e A=a A$ and $A f=A a$. Then there exists a unique element $a^{+} \in A$ such that

(1) $a a^{+}=e$.

(2) $f a^{+}=a^{+}$.

Moreover,

(3) $a^{+} a=f$.

(4) $A e=A a^{+}$.

(5) $f A=a^{+} A$.

(6) $a=a a^{+} a$.

(7) $a^{+}=a^{+} a a^{+}$.

Two idempotents $e$ and $f$ are algebraically equivalent via $a$ and $b(a, b: e \sim f)$ if $e=a b, f=b a, a \in e A f$ and $b \in f A e$. This is easily seen to be an equivalence relation. The idempotents $e$ and $f$ are algebraically equivalent if and only if $A e$ and $A f$ are isomorphic $A$-modules; moreover, in that case, the mapping $x \rightarrow b x a$ is a ring isomorphism of $e A e$ onto $f A f$ [4, pp. 21-23].

Notice that by Lemma 1, if $e A=a A$ and $A f=A a$, then $e$ and $f$ are algebraically equivalent via $a, a^{+}$. This observation enables us to relate algebraic equivalence in $A$ to semi-orthogonal perspectivity in $L(A)$.

THEOREM 2. If $A e \sim A f$, then $e \sim f$.

Proof. Suppose $A g: A e \sim A f$. Put $a=e(1-g)$ and $b=f(1-g)$; then $a$ and $b$ are regular by Theorem 2.1 (2). An easy computation shows $e A=R L(e)=R L(e(1-g))=R L(a)=a A$ and similarly $f A=$ $b A$. Moreover, $A e \oplus A g=A g \oplus A f$ implies $R(a)=R(b)$; thus $A a=$ $L R(a)=L R(b)=A b$. Choose an idempotent $h$ with $A h=A a=A b$. Then by our observation above, $e \sim h$ and $h \sim f$. Hence $e \sim f$.

For semi-orthogonal left ideals, the converse of Theorem 2 is also valid. We prove this as a first consequence of Lemma 2. With $A e \# A f$, this fundamental lemma establishes a bijection of $e A f$ onto, what might be termed, the set of relative semi-orthocomplements of $A f$ in $A e \oplus A f$.

LemMa 2. Let $E=A e$ and $F=A f$ with $E \# F$.

(1) If $G \oplus F=E \oplus F$ with $G \in L(A)$, then $G=A(e-a)$ for some 
unique $a \in e A f$.

(2) If $a \in e A f$, then there exists a left ideal $G \in L(A)$ such that

(i) $G=A(e-a)$.

(ii) $G \oplus F=E \oplus F$.

(iii) $E \vee G=E \oplus L R(a)$.

(iv) $E \cap G=E \cap L(a)$.

Proof. To prove (1), let $g$ be an idempotent generator for $G$. Choose $w$ and $x$ in $A$ such that $e=w g+x f$. Then $e=e w g+e x f$. Put $a=e x f$. Then $e-a=e w g \in G$; so $A(e-a) \leqq G$. Conversely, $g=y e+z f=y(e-a)+y a+z f=y e w g+y a+z f$ for some $y, z \in A$. But $g-y e w g=y a+z f \in G \cap F=(0)$, so that $g=y e w g=y(e-a)$. Hence $G=A g \leqq A(e-a)$.

If also $b \in F=A f$ with $e-b \in G$, then $a-b=(e-b)-(e-a) \epsilon$ $G \cap F=(0)$; so $a=b$. This establishes the uniqueness of $a$.

To prove (2), let $e_{0}$ and $f_{0}$ denote orthogonal idempotent generators for $E$ and $F$ respectively. Put $g=e_{0}-e_{0} a$ and $G=A g$. Since $a e_{0}=$ $a f e_{0}=a f f_{0} e_{0}=0$, we find that $g=g^{2}$. Thus $G \in L(A)$. Now $g=$ $e_{0}(e-a)$ and $e-a=e\left(e_{0}-e_{0} a\right)=e g$ implies $G=A g=A(e-a)$, proving (i). The remaining parts of (2) are straightforward computations.

Theorem 2. Let Ae\#Af. Then Ae $\sim A f$ if and only if $e \sim f$.

Proof. Suppose $a, b: e \sim f$. Put $G=A(e-a)$ and $H=A(f-b)$. Then by Lemma $2(2), G \oplus A f=A e \oplus A f=A e \oplus H$. But $e-a=$ $a b-a=a(b-f)=-a(f-b)$ and $f-b=b a-b=b(a-e)=-b(e-$ $a)$, showing that $G=A(e-a)=A(f-b)=H$. Thus $A e \oplus G=$ $A e \oplus A f=G \oplus A f$.

4. Regularity. In this section, we characterize those Rickart rings $A$ in which $E \cap F=(0)$ implies $E \# F$ for all $E$ and $F$ in $L(A)$. It will be convenient in the two lemmas and in Theorem 1 to adopt some notation. Let $a$ and $b$ denote regular elements with $A e=A a$ and $f A=b A$. Choose $a^{+}$and $b^{+}$by Lemma 3.1 so that $a^{+} a=e$ and $b b^{+}=f$; choose idempotent generators $g$ and $h$ of $L R(a b)$ and $R L(a b)$ respectively. In the context of Rickart *-semigroups, Theorem 1 is due to D. J. Foulis [2].

Lemma 1. If eb or af is regular, then so is $a b$.

Proof. Suppose $e b$ is regular. Choose an idempotent generator $k$ for $A e b$ and choose $(e b)^{+}$so that $(e b)^{+} e b=k$. Put $x=(e b)^{+} a^{+} h$. Then $x a b=(e b)^{+} a^{+} h a b=(e b)^{+} a^{+} a b=(e b)^{+} e b=k$. Then $a b x a b=a b k=(a e) b k=$ $a(e b) k=a(e b)=(a e) b=a b$, showing that $a b$ is regular. The argument for $a f$ is similar. 
Lemma 2. If $a b$ is regular, so are eb and af.

Proof. Choose $(a b)^{+}$so that $a b(a b)^{+}=h$. Let $k$ denote an idempotent generator of $L R(e f)$ and put $x=k b(a b)^{+}$. Then $a f x=a f k b(a b)^{+}=$ $(a e) f k b(a b)^{+}=a(e f) k b(a b)^{+}=a(e f) b(a b)^{+}=(a e) f b(a b)^{+}=a f b(a b)^{+}=$ $a b(a b)^{+}=h$. Hence afxaf $=h a f=h a b b^{+}=a b b^{+}=a f$, showing that $a f$ is regular. Similarly $e b$ is regular.

THEOREM 1. ab is regular if and only if ef is regular.

Proof. If $a b$ is regular, then so is $e b$ by Lemma 2. Since $e b$ is regular, so is ef by Lemma 2 again, applied with $a=e$.

Conversely, if ef is regular, then so is $e b$ by Lemma 1, applied with $a=e$. Then since $e b$ is regular, so is $a b$ by Lemma 1 again.

THEOREM 2. These conditions are equivalent:

(1) ef is regular for every idempotent $e$ and $f$.

(2) If $a$ and $b$ are regular, then so is $a b$.

(3) If $E \cap F=(0)$, then $E \# F$.

Moreover, if $A$ is a matrix ring, we may add

(4) $A$ is a regular ring.

Proof. The equivalence of (1) and (2) is a consequence of Theorem 1. That (1) implies (3) is a consequence of Theorem 2.1 (2). Using the notation of the proof of Corollary 2.1, we may show that (3) implies (1); with $E=A e$ and $F=A f$, we have $A e_{0} \cap A f_{0}=(0)$ as before. Then by (3), $A e_{0} \# A f_{0}$. Consequently, $e_{1}\left(1-f_{1}\right)=e_{0}\left(1-f_{0}\right)$ is regular by Theorem 2.1, and hence $e(1-f)$ is regular. Thus (3) implies $e(1-f)$ is regular for every idempotent $e$ and $f$, and this is evidently equivalent to (1).

Let us now suppose that $A$ is a Rickart matrix ring of order $\geqq 2$. If $A$ is a regular ring, then $E \cap F=(0)$ implies $E \# F$ for all $E$ and $F$ in $\boldsymbol{L}(A)$ by Theorem 2.1. Conversely, if this condition holds for all $E$ and $F$ in $L(A)$, we show that $A$ is a regular ring. To this end, let $e_{i j}, 1 \leqq i, j \leqq n$, be a family of matrix units for $A$. We shall show that $e_{11} A e_{11}$ and hence $A$, which is isomorphic to the $n \times n$ matrix ring over $e_{11} A e_{11}$, is a regular ring.

Let $e_{11} x e_{11}$ denote an arbitrary element in $e_{11} A e_{11}$; put $a=e_{11} x e_{12}$ and choose idempotent generators $e$ and $f$ for $R L(a)$ and $L R(a)$ respectively. Since $R(f)=R(a), a e_{i i}=0$ for $i \neq 2$ implies $f e_{i i}=0$ for $i \neq 2$; since $L(e)=L(a), e_{22} a=0$ implies $e_{22} e=0$. Thus $f e=f\left(\Sigma e_{i i}\right) e=$ $\left(\Sigma f e_{i i}\right) e=\left(f e_{22}\right) e=f\left(e_{22} e\right)=0$, showing that $A e \cap A f=(0)$. Moreover $f(1-e)=f$ is regular. Hence $A e \# A f$. 
Now let $e_{0}$ and $f_{0}$ denote orthogonal idempotents generating $A e$ and Af respectively. Put $g=e_{0}-e_{0} a$. Then, as in the proof of Lemma 3.2, $a=e(1-g)$ and $A g=A(e-a)$. Thus $A e \cap A g=A e \cap L(a)=$ $A e \cap L(e)=(0)$. Then by hypothesis, $A e \# A g$. But this means that $a=e(1-g)$ is regular in $A$. Choose an element $b$ in $A$ with $a b a=a$. Then

$$
\left(e_{11} x e_{12}\right) b\left(e_{11} x e_{12}\right)=a b a=a=e_{11} x e_{12}
$$

or equivalently

$$
\left(e_{11} x e_{12}\right) b\left(e_{11} x e_{11}\right)=e_{11} x e_{11} \text {. }
$$

Thus

$$
\left(e_{11} x e_{11}\right)\left(e_{12} b e_{11}\right)\left(e_{11} x e_{11}\right)=e_{11} x e_{11},
$$

showing that $e_{11} x e_{11}$ is a regular element of $e_{11} A e_{11}$.

Hence $e_{11} A e_{11}$ is a regular ring.

Recall that two left ideals in a von Neumann algebra $A$ are semiorthogonal if and only if their unique generating projections are nonasymptotic. Therefore, a von Neumann matrix algebra with no asymptotic pairs of projections must be regular and hence finite dimensional [8, pp. 85-87]. The definitive result in the general case is due to D. M. Topping [9]. Topping shows that in a von Neumann algebra these conditions are equivalent: (1) $A$ has no asymptotic pairs of projections; (2) $A$ contains no infinite orthogonal sequence of non-abelian projections; (3) $A$ is the direct sum of an abelian subalgebra and a finite dimensional subalgebra. As a consequence of this result, a type $I I_{1}$ von Neumann algebra may contain asymptotic pairs of projections, although its projection lattice is necessarily modular. Thus semi-orthogonality and dual modularity are in general distinct concepts. Using Foulis' characterization of dual modularity in terms of range-closedness, this same example shows that the product of two projections in a von Neumann algebra may have a closed range without being *-regular.

A simple proof, in the spirit of this paper, of (1) implies (2) in Baer *-rings would be worthwhile; for this would show that a complete *-regular ring can contain no infinite orthogonal sequence of non-abelian projections and hence no infinite orthogonal sequence of equivalent projections. A complete *-regular ring must, therefore, be of finite type. This is a difficult step in Irving Kaplansky's proof [3] that an orthocomplemented complete modular lattice is a continuous geometry.

\section{REFERENCES}

1. J. Feldman, Isomorphisms of rings of operators, Thesis, University of Chicago, 1954. 
2. D. J. Foulis, Relative inverses in Baer *-semigroups, Mich. Math. J., 10 (1963), 6584.

3. I. Kaplansky, Any orthocomplemented complete modular lattice is a continuous geometry, Ann. of Math., 61 (1955), 524-541.

4. ㄴ. Rings of Operators, New York: W. A. Benjamin, Inc., 1968.

5. G. W. Mackey, On infinite dimensional linear spaces, Trans. Amer. Math. Soc., 57 (1945), 155-207.

6. S. Maeda, On relatively semi-orthocomplemented lattices, J. Sci. Hiroshima Univ., $24 \mathbf{A}$ (1960), 155-161.

7. D. D. Miller and A. H. Clifford, Regular D-classes in semigroups, Trans. Amer. Math. Soc., 82 (1956), 270-280.

8. J. von Neumann, Continuous Geometry, Princeton: Princeton Univ. Press, 1960.

9. D. M. Topping, Asymptoticity and semimodularity in projection lattices, Pacific. J. Math., 20 (1967), 317-325.

Received October 23, 1970.

UNIVERSITY OF MASSACHUSETTS

AND

KANSAS STATE UNIVERSITY 


\section{PACIFIC JOURNAL OF MATHEMATICS}

\section{EDITORS}

H. SAMELSON

Stanford University

Stanford, California 94305

C. R. HobBY

University of Washington

Seattle, Washington 98105
J. DugundjI

Department of Mathematics

University of Southern California

Los Angeles, California 90007

RICHARD ARENS

University of California

Los Angeles, California 90024

\section{ASSOCIATE EDITORS}

E. F. BECKENBACH

B. H. NeUMaNN

F. WOLF

K. YoshidA

\section{SUPPORTING INSTITUTIONS}

UNIVERSITY OF BRITISH COLUMBIA

CALIFORNIA INSTITUTE OF TECHNOLOGY

UNIVERSITY OF CALIFORNIA

MONTANA STATE UNIVERSITY

UNIVERSITY OF NEVADA

NEW MEXICO STATE UNIVERSITY

OREGON STATE UNIVERSITY

UNIVERSITY OF OREGON

OSAKA UNIVERSITY
UNIVERSITY OF SOUTHERN CALIFORNIA STANFORD UNIVERSITY

UNIVERSITY OF TOKYO

UNIVERSITY OF UTAH

WASHINGTON STATE UNIVERSITY UNIVERSITY OF WASHINGTON

$\stackrel{*}{*} \stackrel{*}{*} \stackrel{*}{*}$ AMERICAN MATHEMATICAL SOCIETY
NAVAL WEAPONS CENTER 


\section{Pacific Journal of Mathematics}

Vol. 39 , No. 1

May, 1971

Charles A. Akemann, A Gelfand representation theory for $C^{*}$-algebras ....

Sorrell Berman, Spectral theory for a first-order symmetric system of

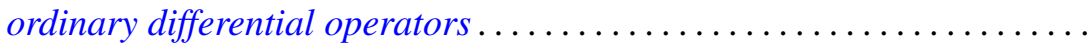

Robert L. Bernhardt, III, On splitting in hereditary torsion theories ........

J. L. Brenner, Geršgorin theorems, regularity theorems, and bounds for determinants of partitioned matrices. II. Some determinantal identities ..........................................

Robert Morgan Brooks, On representing $F^{*}$-algebras .............. 51

Lawrence Gerald Brown, Extensions of topological groups........... 71

Arnold Barry Calica, Reversible homeomorphisms of the real line ........ 79

J. T. Chambers and Shinnosuke Oharu, Semi-groups of local Lipschitzians in

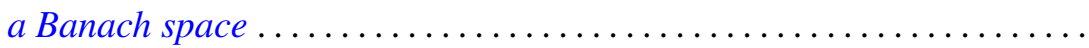

Thomas J. Cheatham, Finite dimensional torsion free rings .............

Byron C. Drachman and David Paul Kraines, A duality between

transpotence elements and Massey products ...................

Richard D. Duncan, Integral representation of excessive functions of a

Markov process ......................................

George A. Elliott, An extension of some results of Takesaki in the reduction

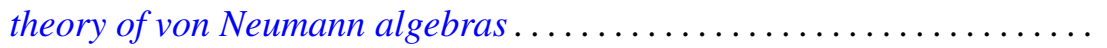

Peter C. Fishburn and Joel Spencer, Directed graphs as unions of partial

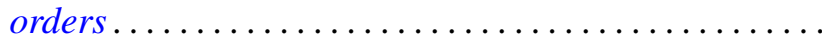

Howard Edwin Gorman, Zero divisors in differential rings ...

Maurice Heins, A note on the Löwner differential equations...

Louis Melvin Herman, Semi-orthogonality in Rickart rings. .

David Jacobson and Kenneth S. Williams, On the solution of linear G.C.D.

equations

Michael Joseph Kallaher, On rank 3 projective planes ... . .

Donald Paul Minassian, On solvable $O^{*}$-groups ...........

Nils Øvrelid, Generators of the maximal ideals of $A(\bar{D})$

Mohan S. Putcha and Julian Weissglass, A semilattice decomposition into

semigroups having at most one idempotent ............

Robert Raphael, Rings of quotients and $\pi$-regularity ....

J. A. Siddiqi, Infinite matrices summing every almost periodic sequence. .

Raymond Earl Smithson, Uniform convergence for multifunctions ...

Thomas Paul Whaley, Mulitplicity type and congruence relations in

universal algebras...

Roger Allen Wiegand, Globalization theorems for locally finitely generated modules... 